\title{
Constraint preserving boundary conditions for the Ideal Newtonian MHD equations
}

\author{
Mariana Cécere ${ }^{1}$, Luis Lehner ${ }^{2}$ and Oscar Reula ${ }^{1}$ \\ ${ }^{1}$ FaMAF, Universidad Nacional de Córdoba, Córdoba, 5000 (Argentina) \\ 2 Department of Physics and Astronomy, Louisiana State University, Baton Rouge, LA 70803-4001
}

\section{Introduction}

Magnetic fields play an important role in the behavior of plasmas and are thought mediate important effects like dynamos in the core of planets and the formation of jets in active galactic nuclei and gamma ray bursts; induce a variety of magnetic instabilities; realize solar flares, etc. (see e.g. [12]). Understanding the role of magnetic fields in these and other phenomena have spurred through the years many efforts to obtain solutions of the magnetohydrodynamic equations. The non-linear nature of these equations limits the understanding that can be gained in a particular problem via analytical techniques. This implies that solutions for complex systems must be obtained by numerical means and a suitable numerical implementation must be constructed for this purpose. Such implementation must be able to evolve the solution to the future of some initial configuration and guarantee its quality. A delicate, subsidiary quantity, can be monitored in part to estimate this. This quantity is the "monopole constraint" $\partial_{i} B^{i}$ which must be zero at the analytical level for a consistent solution. This quantity is not a part of the main variables, rather it is a derived quantity which should be satisfied by a true physical solution. In practice, unless a numerical implementation of the MHD equations is carefully designed this constraint can be severely violated. This, in turn, signals (and is sometimes the cause) of a degrading numerical solution. For these reasons, several approaches have been investigated and developed for guaranteeing a controlled behavior of this quantity. One such approach is known as the constraint transport technique [3,4] which adopts a particular algorithm that staggers the variables appropriately to ensure the satisfaction of the constraint at round-off level within Finite Difference and Finite Elements techniques. This approach has been quite successful in a number of applications across different disciplines and particularly relevant in astrophysics applications $[5,6,7,8,9,10,11,12]$. However, by design it imposes limits on the algorithmic options available to an implementation. This fact can be at odds, or introduce complications, with applications where adaptive mesh refinement is required and/or advanced numerical techniques (that exploit useful properties of the equations) are adopted. An alternative approach, which controls the constraint at truncation-error level maintains complete freedom in the numerical techniques to be adopted. This approach, referred to as divergence cleaning puts the burden to control the constraint not on the algorithm to be employed 
but rather on the system of equations to be solved itself [13,14. This is achieved by considering an additional variable suitably coupled to the system through another equation so that, through the evolution, the constraint behavior is kept under control. While this method has, to date, received less attention than the constraint transport one, successful applications in diverse scenarios have already illustrated its usefulness (e.g. [14]15,4],16,17]).

Regardless of the technique employed, boundary conditions can play a significant role and may spoil all efforts towards a correct implementation in the absence of boundaries. Clearly, even when a stable option is adopted, it need not be consistent with the goal of preserving the constraint (be it at round-off or truncation levels) and so carefully designed boundary conditions must be formulated. Commonly employed options include straightforward outflowtype conditions - which do not enforce the constraint- or "absorbing" boundary conditions which aim to reduce the influence of spurious effects induced at the boundary in the numerical solution [18].

In the present work we concentrate on formulating constraint preserving boundary conditions for the Newtonian ideal MHD equations (for systems with and without divergence cleaning). Such a task is intimately related to the hyperbolic properties of the equations and so we re-analyze the system of equations and discuss alternatives for controlling the constraints through the divergence cleaning technique.

We therefore organize our presentation along the following lines. In section II we analyze the system of equations and the formulation of boundary conditions beginning with a simplified model from which we draw the strategy to apply in the complete MHD system. Section III presents a series of tests that highlight the benefits gained by our construction. We conclude in section IV with some final comments.

\section{The Newtonian equations \& constraint hyperbolic cleaning}

The equations describing the ideal Newtonian MHD equations in terms of the variables $\left(\rho, p, v^{i}, B^{i}\right)$ are [1,2]):

$$
\begin{aligned}
\partial_{t} \rho & =-\nabla_{i}\left(\rho v^{i}\right) \\
\partial_{t} p & =-v^{i} \nabla_{i} p-\gamma p \nabla_{j} v^{j} \\
\rho \partial_{t} v^{i} & =-\rho v^{j} \nabla_{j} v^{i}-\nabla^{i} p-\nabla_{j}\left(e^{i j} \frac{1}{2} B_{k} B^{k}-B^{j} B^{i}\right) \\
\partial_{t} B^{i} & =-\nabla_{j}\left(v^{j} B^{i}-v^{i} B^{j}\right) .
\end{aligned}
$$

In this form, this system of equations is weakly hyperbolic since there is no complete set of eigenvectors. This indicates instabilities are likely to arise unless some modes are carefully controlled. The constraint transport technique attempts to do so at the algorithmical level by enforcing $\partial_{i} B^{i}=0$ at the discrete level. We here choose an alternative approach where the situation is remedied at the analytical level through the addition of an extra field coupled to the system in a suitable way [1314]. To simplify the discussion, we first consider a simpler model which captures essential features of the main system. 


\subsection{Preliminaries}

To illustrate this approach consider first the system obtained when the fluid field variables as given and stationary. The resulting system describes a simple model that shares key problematic features of the original ideal MHD system (1), although in the latter case additional aspects enter under consideration. In this simple case one just has the magnetic field which evolves under,

$\partial_{t} B_{i}=-\nabla_{j}\left(v^{j} B^{i}-v^{i} B^{j}\right)$.

This equation is already weakly hyperbolic, since a plane-wave analysis indicates that when the wave number vector is perpendicular to the velocity vector there exists only two linearly independent eigenvectors. To see this consider the case where the wave number vector is normal to the velocity vector, and define coordinates axis such that the first axis lies along the wave vector -so $k_{i}=(k, 0,0)$ - the second along the velocity vector, - so $v^{i}=(0, v, 0)$ - and the third perpendicular to both of them. Then the eigenvalue-eigenvector problem, obtained by expressing $B^{i}=U e^{\sigma t+k_{i} x^{i}}$, gives rise to the following problem: $\sigma U=M U$ (with $\sigma$ and $U$ a frequency and a vector to be determined) and $M$ defined as

$M=\left(\begin{array}{ccc}0 & 0 & 0 \\ k v & 0 & 0 \\ 0 & 0 & 0\end{array}\right)$

which is clearly non diagonalizable. Thus, there is no stability in the usual $L^{2}$ norm sense. Interestingly the divergence of the magnetic field is preserved by equation (11), that is, if one defines $D:=\nabla_{k} B^{k}$ and takes the divergence of (1) one obtains the induced evolution equation for $D$ as $\partial_{t} D=0$. Hence, if $D$ is zero initially it remains so as along as the time integration lines do not intersect a boundary. However, we note that if instead of considering the $L_{2}$ norm, one considers a different norm defined by adding a term proportional to the divergence of $B_{i}$, namely a norm of the type:

$\mathcal{E}:=\int_{\Sigma_{t}}\left[B^{2}+c\left(\nabla_{k} B^{k}\right)^{2}\right] d V$

one can show that $\dot{\mathcal{E}}=0$ and so this energy is controlled. Therefore, from an analytical point of view equation (1) is not a bad one.

At the numerical level, things become more delicate as generic violations of this constraint will arise due to truncation or round-off errors which might grow unless a careful implementation of the equations is adopted. Furthermore, even when an integration scheme is available that controls the constraint in the absence of boundaries, (which is not difficult to obtain in flat space in Cartesian coordinates - which we use in this work-), the boundary conditions modify the equations at the boundaries which can cause the constraint to grow and propagate to the interior. Thus, we next discuss a way to achieve a more robust behavior. Rather than doing so by the particular numerical algorithm employed we work first at the analytical level and adopt a strategy that would help even beyond the simple problem adopted here. 
To remedy the lack of strong hyperbolicity and good constraint propagation a possible approach can be adopted which makes use of the freedom to add to equation (11) any term proportional to that divergence. The proposed system is (in analogy with that in [14]):

$$
\begin{aligned}
\partial_{t} B_{i} & =-\nabla_{j}\left(v^{j} B^{i}-v^{i} B^{j}\right)-c_{l} \nabla_{i} \phi, \\
\partial_{t} \phi & =-c_{l} \nabla_{k} B^{k}-s \phi .
\end{aligned}
$$

Notice that for $\phi=0$ the modified system is equivalent to the original one, and $\phi$ has as a source proportional to the constraint. Thus if boundary and initial data are such as to guarantee that $\nabla_{k} B^{k}=0$, and trivial data is given for $\phi$ the solution to the modified system is also a solution of the original equation. The advantage of this modification is that now the system is strongly hyperbolic, so it has a well posed initial problem and is stable irrespective of whether or not $\nabla_{k} B^{k}$ vanishes. Furthermore, the induced evolution equation for $D$ is no longer trivial, and it implies that the field $D$ propagates with speed $c_{l}$. Consequently one can make the constraint violations to propagate away from the integration region, and, if correct boundary conditions are given, leave the computational region entirely. Additionally, the last term in the equation for $\phi$ in (3) induces a decay of the constraint for $s>0$ as it travels along the integration region further helping to keep it under control.

Drawing from this exercise, other modifications are certainly possible; for instance, one could consider a more complex system given by,

$$
\begin{aligned}
\partial_{t} B_{i} & =-u^{j} \nabla_{j} B_{i}+B^{j} \nabla_{j} u_{i}-B_{i} \nabla_{j} u^{j}+(1-\alpha) u^{i} \nabla_{j} B^{j}-c_{l} \nabla_{i} \phi \\
\partial_{t} \phi & =-\beta u^{j} \nabla_{j} \phi-c_{l} \nabla^{i} B_{i}-s \phi .
\end{aligned}
$$

Which, for a range of values of the parameters $\alpha$ and $\beta$ for is strongly hyperbolic. Among these, of particular interest is the system with $\alpha=\beta=1$, which is strongly hyperbolic even in the limit $c_{l} \rightarrow 0$, so its associated initial value problem would be well posed even without the coupling to the field $\phi$. Another interesting choice is the one $\alpha=\beta=0$ since the resulting system can be expressed in conservative form. This property is often preferred in applications where shocks are present as one can easily take advantage of special algorithms defined to deal with these issues (see e.g. [19,20,21,22,23]).

Boundary conditions To fix ideas, we now study the possible boundary conditions for the above system (with $\alpha=\beta=0$ ). Our goal is to define conditions which can yield a well posed problem and, if possible, guarantee no violations are introduced into the computational domain. To this end we must determine which are the incoming and outgoing modes off the boundary surface as this information is key to understand what data is freely specifiable. For this we seek a solution of the form $\left(B^{i}, \phi\right)=U e^{\sigma t+n_{i} x^{i}}$, where $U$ is a vector to be determined along with the frequency $\sigma$, and $n_{i}$ is the outgoing normal to the boundary under consideration. Applying this solution to the system we obtain:

$$
\begin{aligned}
\sigma B^{i} & =-B^{i} v_{n}+v^{i} B_{n}-c_{l} n^{i} \phi, \\
\sigma \phi & =-c_{l} B_{n},
\end{aligned}
$$


where $B_{n}:=B^{i} n_{i}$, and $v_{n}:=v^{i} n_{i}$. This problem of eigenvalues/eigenvectors can be solved to determine the incoming $(\sigma>0)$, tangential $(\sigma=0)$ and outgoing $(\sigma<0)$ modes. A necessary condition for a well posed problem for hyperbolic systems indicates that data must be given only to the incoming modes, since the others are determined from the inside of the integration region [25,24]. Recall that the incoming modes can be defined even as linear functions of the outgoing ones so long as the coefficients are small enough.

At a given boundary, there are three eigenvalues, $\left(\sigma_{0}:=-v_{n}, \sigma_{ \pm}:= \pm c_{l}\right)$. The first corresponds to two linearly independent modes which span the tangent space of the boundary point (i.e. they are perpendicular to $n_{i}$ ). They are positive whenever the velocity is incoming, these are the modes which are dragged along $v^{i}$. The other eigenvalues correspond to the cases where we can choose $B_{n}:=b$ arbitrarily and,

$$
\begin{aligned}
\phi & =\mp b, \\
B^{i} & =\frac{\left(v^{i} \pm n^{i} c_{l}\right) b}{v_{n} \pm c_{l}} .
\end{aligned}
$$

Notice one can always adopt a value for $c_{l}$ larger than the maximum velocity expected so that denominator is never zero. The eigenvector corresponding to the positive eigenvalue determine the combination of variable that must be always suitably given to preserve the constraint. The complete expression for the eigenbase and its co-base is.

$$
\begin{aligned}
U_{1}^{0} & :=\left(0, e_{1}, 0\right) \quad, \quad \sigma=-v_{n}, \\
U_{2}^{0} & :=\left(0, e_{2}, 0\right) \quad, \quad \sigma=-v_{n}, \\
U^{ \pm} & :=\left(1, \frac{\left(v^{i} \pm n^{i} c_{l}\right)}{v_{n} \pm c_{l}}, \mp 1\right) \quad, \quad \sigma= \pm c_{l},
\end{aligned}
$$

where the generic vector is $U:=\left(B_{n}, \tilde{B}^{i}, \phi\right)$ with $\tilde{B}^{i} n_{i}=0$, and $\left\{e_{1}, e_{2}\right\}$ are two orthonormal vectors on the tangent of the boundary.

The co-base is given by

$$
\begin{aligned}
\theta_{0}^{1} & :=\left(\frac{v_{n} v_{1}}{c_{l}^{2}-v_{n}^{2}}, e_{1}, \frac{c_{l} v_{1}}{c_{l}^{2}-v_{n}^{2}}\right), \\
\theta_{0}^{2} & :=\left(\frac{v_{n} v_{2}}{c_{l}^{2}-v_{n}^{2}}, e_{2}, \frac{c_{l} v_{2}}{c_{l}^{2}-v_{n}^{2}}\right), \\
\theta_{ \pm} & :=\frac{1}{2}(1,0, \mp 1),
\end{aligned}
$$

where $v_{1}:=e_{1}^{i} v_{i}$, and $v_{2}:=e_{2}^{i} v_{i}$. In order to see how we must define boundary values consistent with the constraints we now analyze the subsidiary system determining the evolution of the constraint. In order to treat it as a first order system we define a new variable $\delta_{i}:=\nabla_{i} \phi$, whose evolution equation is determined by (the time derivative of) the evolution equation of $\phi$. The full subsidiary system is then:

$\partial_{t} D=-c_{l} \nabla^{i} \delta_{i}$

$\partial_{t} \delta_{i}=-c_{l} \nabla_{i} D-s \delta_{i}$.

Here again we must investigate now which are the incoming and outgoing modes for this system. If we can impose boundary conditions to the main system so that we ensure no incoming mode 
is created for this subsidiary system, then uniqueness of the (trivial) solution would guarantee that the solution of the main system has vanishing constraint quantities and so it is a solution of the original system. As done previously, plane wave solutions of the above system perpendicular to the boundary give rise to the following eigenvalue/eigenvector problem:

$\kappa D=-c_{l} n^{i} \delta_{i}$

$\kappa \delta_{i}=-c_{l} n_{i} D$,

and its eigenvalues are $\left\{\kappa_{0}=0, \kappa_{ \pm}= \pm c_{l}\right\}$ with the corresponding eigenvectors:

$$
\begin{aligned}
V_{1}^{0} & :=\left(0, e_{1}, 0\right) \quad, \quad \kappa_{0}=0, \\
V_{2}^{0} & :=\left(0, e_{2}, 0\right) \quad, \quad \kappa_{0}=0, \\
V^{ \pm} & :=\left(1, \frac{\left(v^{i} \pm n^{i} c_{l}\right)}{v_{n} \pm c_{l}}, \mp 1\right) \quad, \quad \kappa_{ \pm}= \pm c_{l},
\end{aligned}
$$

where the generic vector is $V:=\left(\delta_{n}, \tilde{\delta}^{i}, D\right)$ with $\tilde{\delta}^{i} n_{i}=0$, and $\left\{e_{1}, e_{2}\right\}$ are two orthonormal vectors tangent to the boundary.

The co-base is given by

$$
\begin{aligned}
\Theta_{0}^{1} & :=\left(0, e_{1}, 0\right), \\
\Theta_{0}^{2} & :=\left(0, e_{2}, 0\right), \\
\Theta_{ \pm} & :=\frac{1}{2}(1,0, \mp 1) .
\end{aligned}
$$

Here again the theory of boundary conditions indicate we should give as a boundary condition the incoming mode as a linear function of the outgoing one. That is we should ensure that the boundary data for the main system is such that:

$\Theta_{+}(V)+a \Theta_{-}(V) \hat{=} 0 \quad, \quad|a|<1$

This is a linear combination of space derivatives of the fields and can be implemented in several different ways depending on the problem at hand. Here we choose to implement it at the level of the evolution equations, namely by modifying the evolution equations at the boundary so that the constraint is satisfied there. From the evolution equations of the main system we can solve for $D$ and $\delta_{n}$ in terms of the time derivatives of $B_{n}$ and $\phi$ (and the remaining equations). Hence

$$
\begin{gathered}
\delta_{n}=\frac{-1}{c_{l}}\left(\partial_{t} B_{n}-F_{n}\right), \\
D=\frac{-1}{c_{l}}\left(\partial_{t} \phi-F_{\phi}\right),
\end{gathered}
$$

with $F_{n}=-n_{i} \partial_{j}\left(u^{j} B^{i}-u^{i} B^{j}\right)$, and $F_{\phi}=-s \phi$.

We can then translate the above boundary condition into:

$$
-\dot{B}_{n}(1+a)+\dot{\phi}(1-a)+F_{n}(1+a)-F_{\phi}(1-a) \hat{=} 0,
$$

which can be re-expressed as:

$$
-2\left(\theta_{+}(U)+a \theta_{-}(U)\right)+F_{n}(1+a)-F_{\phi}(1-a) \hat{=} 0 .
$$


For the particular case of setting the incoming constraint modes to zero $(a=0)$ this implies,

$\theta_{+}(U) \hat{=} \frac{1}{2}\left(F_{\phi}-F_{n}\right)$

Thus, the boundary condition defined by the equation above is not only maximally dissipative but also enforces the constraint.

\subsection{The complete system}

We now turn our attention to the complete system and develop an analogous strategy following the discussion considered above. In most cases the literature on the subject considers the MHD system expressed in terms variables $U=\left(\rho, e, u^{i}, B^{i}\right)$ rather than $U=\left(\rho, p, u^{i}, B^{i}\right)$ -ie the internal energy $e$ instead of the pressure $p$-. While this involves a simple change of variables it turns out the characteristic decomposition is simpler in the latter case. So, our evolution system will be given by the former, and we will obtain the characteristic structure for the latter followed by a straightforward change of variables. Our system of interest is a variation of the initial MHD equations as (see [14]):

$$
\begin{aligned}
\partial_{t} \rho & =-\nabla_{i}\left(\rho v^{i}\right), \\
\rho \partial_{t} v^{i} & =-\rho v^{j} \nabla_{j} v^{i}-\nabla^{i} p-B_{k}\left(\nabla^{i} B^{k}-\nabla^{k} B^{i}\right)-\alpha B^{i} \nabla_{k} B^{k}, \\
\partial_{t} B^{i} & =-\nabla_{j}\left(u^{j} B^{i}-u^{i} B^{j}\right)-\alpha u^{i} \nabla_{j} B^{j}-c_{l} \nabla^{i} \phi, \\
\partial_{t} e & =-\nabla_{i}\left(\left(e+p+\frac{1}{2} B^{2}\right) v^{i}-B^{i} v \cdot B\right)-\alpha v^{i} B_{i} \nabla_{k} B^{k}-c_{l} B^{k} \nabla_{k} \phi, \\
\partial_{t} \phi & =-\alpha u^{j} \nabla_{j} \phi-c_{l} \nabla_{j} B^{j}-s \phi,
\end{aligned}
$$

where

$p:=(\gamma-1)\left(e-\frac{1}{2} \rho v^{2}-\frac{1}{2} B^{2}\right) \quad, \quad c_{s}^{2}:=\frac{\gamma p}{\rho}$

Thys system includes both the possibility of divergence cleaning $\left(c_{l} \neq 0\right)$ and Galilean invariance $(\alpha=1)$ and is strongly hyperbolic, hence has a complete set of eigenvectors. This will allow us to introduce a new boundary treatment which ensures no constraint violations are introduced through the computational domain boundaries. The parameter $\alpha$ controls the freedom of adding the constraint equation. In what follows, we shall use the values $\alpha=0,1$. The value $\alpha=0$ corresponds to the purely conservative system (notice that velocity equation can be re-written with the help of the first equation as momentum conservation). The case with $\alpha=1$ gives rise to a system that is Galilean invariant (see [14]) and also to the case where the system is strongly hyperbolic [26,27] irrespective of the field $\phi$. Hence one can study the limit where the $\phi$ field decouples and the constraint propagates along the velocity field.

Boundary system As done previously, we now consider a boundary with outgoing unit normal $n^{i}$ and obtain the characteristic decomposition at this boundary. For notational purposes we will employ an overbar to denote the perturbation on a given variable, for instance $\bar{\rho}$ will 
denote the perturbation of $\rho$ which will be considered as a fixed background quantity. The characteristic decomposition is then determined from the system,

$$
\begin{aligned}
\sigma \bar{\rho} & =-v_{n} \bar{\rho}-\rho \bar{v}_{n} \\
\sigma \bar{v}_{i} & =-v_{n} \bar{v}_{i}+B_{i} \bar{B}_{n} / \rho+\left(B_{n} / \rho\right) \bar{B}_{i}-n_{i}\left(B_{j} \bar{B}^{j}+\bar{p}\right) / \rho, \\
\sigma \bar{B}_{i} & =-v_{n} \bar{B}_{i}+v_{i} \bar{B}_{n}-B_{i} \bar{v}_{n}+B_{n} \bar{v}_{i}+n_{i} \bar{\phi} \\
\sigma \bar{p} & =-c_{s}^{2} \rho \bar{v}_{n}-(\gamma-1) B_{j} v^{j} \bar{B}_{n}-v_{n} \bar{p}+v(1-\gamma) B_{n} \bar{\phi} . \\
\sigma \bar{\phi} & =c_{l}^{2} \bar{B}_{n}
\end{aligned}
$$

The solution to this system is cumbersome and requires dealing with different cases. The full analysis and solution is presented in appendix $\mathrm{A}$ and expressed in terms of a vector whose entries are: $U=\left(\bar{\rho}, \bar{v}_{n}, \tilde{\bar{v}}_{i}, \bar{B}_{n}, \tilde{\bar{B}}_{i}, \bar{p}, \bar{\phi}\right)^{T}$ indicating perturbations off $\left(\rho, v^{i} n_{i}, \tilde{v}^{i}, B^{i} n_{i}, \tilde{B}^{i}, p, \phi\right)^{T}$ respectively with $\tilde{v}^{i}, \tilde{B}^{i}$ indicating the components of $v^{i}$ and $B^{i}$ orthogonal to the boundary. While the full characteristic decomposition is lengthy, the characteristic decomposition associated to $B_{n}$ and $\phi$ is particularly simple. In fact, the associated co-basis is given by

$\Theta_{L \pm}=\frac{1}{2 B_{n}}\left(0,0,0,1,0,0, \pm c_{l}^{-1}\right)$,

i.e. involving essentially just $B_{n}$ and $\phi$, hence the imposition of suitable boundary conditions will be directly related to that of our discussion of the simplified model in section 2.1 .

\subsection{Boundary conditions}

Having the complete characteristic structure it is now rather straightforward to determine the possible boundary conditions. A simple one is to set all incoming modes to zero but this, generically, will not be consistent with the constraint. To this end, as explained in section 2.1, one must analyze the induced evolution for the constraint and obtain from it a recipe for what to provide to the incoming modes. In the general case discussed here, notice that $\Theta_{L \pm}$ is essentially the same that gives rise to equation (12) since is non-trivial only for the $B_{n}$ and $\lambda$ components. As a result, one can follow the same strategy and formulate constraint preserving boundary conditions by enforcing

$\dot{U}=U L_{+}\left(\frac{1}{2}\left(F_{\phi}-F_{n}\right)\right) \equiv £(U)$,

where $£(U)$ denotes the (maximally dissipative) constraint preserving boundary condition defined by equation (17).

\section{Numerical Tests}

In this section we present the results of tests aimed to examine the solution's behavior with the different possible choices. To this end we constructed an implementation of the MHD equations (eqns. 13) on a 2-dimensional domain with Cartesian coordinates $(x, y) \in[-L, L] \times$ $[-L, L]$. To discretize the system we employ a set of techniques which guarantee the stability of generic linear hyperbolic systems. These techniques are constructed to satisfy at the discrete level, the conditions holding at the analytical one to prove well posedness of a problem through 
energy estimates [24]. Thus we adopt discrete derivative operators satisfying summation by parts, a 3rd order Runge-Kutta time integration and add dissipation through a Kreiss-Oliger type operator consistent with summation by parts. Finally, in all the tests considered we add a homogeneous 'atmosphere' throughout the computational domain in the initial data (and a 'floor' during the evolution) for the density and the pressure to prevent negative values from arising in the simulation. We typically adjust the atmosphere (floor) values to be $10^{-4}\left(10^{-6}\right.$ ) times the maximum of the initial values of $\rho$ and $e$ respectively. Notice that this straightforward implementation is not expected to handle shocks or discontinuities, however, since our goal is to examine boundary conditions we restrict here to scenarios where these effects do not arise within the time of interest.

For testing purposes we adopt a few different initial scenarios which are based on modifications of two commonly employed initial data sets:

Rotor test: This is a variation of the MHD Rotor problem [28] which is smooth and allows for considering an additional flow field and initial constraint violation,

$\rho(x, y)= \begin{cases}10 & \text { if } r \leq r_{0} \\ 1+9 f(r) & \text { if } r_{0}<r<r_{1} \\ 1 & \text { if } r_{1} \leq r\end{cases}$

$v^{x}(x, y)=\nu^{x}+ \begin{cases}-f(r) v_{o} \frac{y}{r_{0}} & \text { if } r \leq r_{0} \\ -f(r) v_{o} \frac{y}{r} & \text { if } r_{0}<r<r_{1} \\ 0 & \text { if } r_{1} \leq r\end{cases}$

$v^{y}(x, y)=\nu^{y}+ \begin{cases}f(r) v_{o} \frac{x}{r_{0}} & \text { if } r \leq r_{0} \\ f(r) v_{o} \frac{x}{r} & \text { if } r_{0}<r<r_{1} \\ 0 & \text { if } r_{1} \leq r\end{cases}$

and

$$
\begin{aligned}
p & =1 \\
B^{x} & =B_{0}+\mathcal{B}^{x} \\
B^{y} & =0 \\
\phi & =0
\end{aligned}
$$

with $r_{0}=1, r_{1}=2, r=\sqrt{x^{2}+y^{2}}, \gamma=1.4, v_{0}$ a constant and $f(r)$ an interpolating function. For the general tests we adopt

$f(r)=\frac{\left(r_{1}-r\right)}{\left(r_{1}-r_{0}\right)}$.

However, for the convergence test we adopt a smooth interpolating polynomial and velocity profiles as 


$$
\begin{aligned}
f(r) & =P_{5}(r) \\
v_{o} & =\tilde{v}_{o} r^{4} /(1+r)^{4}
\end{aligned}
$$

with $P_{5}(r)$ a polynomial of order 5 such that $P_{5}\left(r_{0}\right)=1 ; P_{5}\left(r_{1}\right)=0$ and both first and second derivatives at $r=\left\{r_{0}, r_{1}\right\}$ are zero. Thus, the corresponding initial data is at least $C^{2}$ throughout the computational domain.

Finally, the functions $\left(\nu^{x}, \nu^{y}\right)$ and $\mathcal{B}^{x}$ are included to consider further modifications for specific tests, in particular choosing :

$-\mathcal{B}^{x}=\kappa e^{-r^{2}}$ allows one to introduce data violating the constraints.

- $\left(\nu^{x}, \nu^{y}\right)=-\epsilon\left(1-e^{-r^{2}}\right)(x, y)$ defines data inflowing to further test boundary issues.

Blast test: This is a variation of the MHD Blast problem [29].

The initial data is given by,

$$
\begin{aligned}
\rho & =1.0 \\
v_{x} & =0.0 \\
v_{y} & =0.0 \\
B_{x} & =4.0 \\
B_{y} & =0.0 \\
p & =e^{-r^{2} / 2} \\
\phi & =0.0
\end{aligned}
$$

Additionally, we also studied the solution's behavior employing slight variations of this data set. In particular, cases with initial incoming or outgoing velocity fields at points of the boundary. In this way we could test different modes on the boundary which become in some cases outgoing or incoming according to the velocities chosen.

Boundary conditions adopted For boundary conditions we adopt one of the following three possible cases:

(i) Freezing boundary conditions. Defined by $\dot{U}_{i}=0$, for $i=1 . .7$ (denoted by FR).

(ii) Outflow boundary condition (incoming modes set to zero). Defined as $\Xi^{+} \dot{U}=0$, with $\Xi^{+}=\sum_{i} U_{j}^{+} \theta_{j}^{+}$, for $j$ such that $\lambda_{j}>0$ (denoted by NI).

(iii) Constraint preserving boundary condition. Defined as $\Xi^{+} \dot{U}=£(U)$, as defined by eqn. (17) (denoted by CP).

in all tests, and compare the solutions' behavior when employing each option. We begin by considering the flux-conservative form of the equations (keeping $\alpha=0$ ) and then examine particular cases with the Galilean invariant form $(\alpha=1)$.

\subsection{Testing the implementation}

In the first test we confirm the overall convergent behavior of the numerical solution when considering sufficiently smooth initial data. We evolve the $C^{2}$ version of the Rotor initial data 


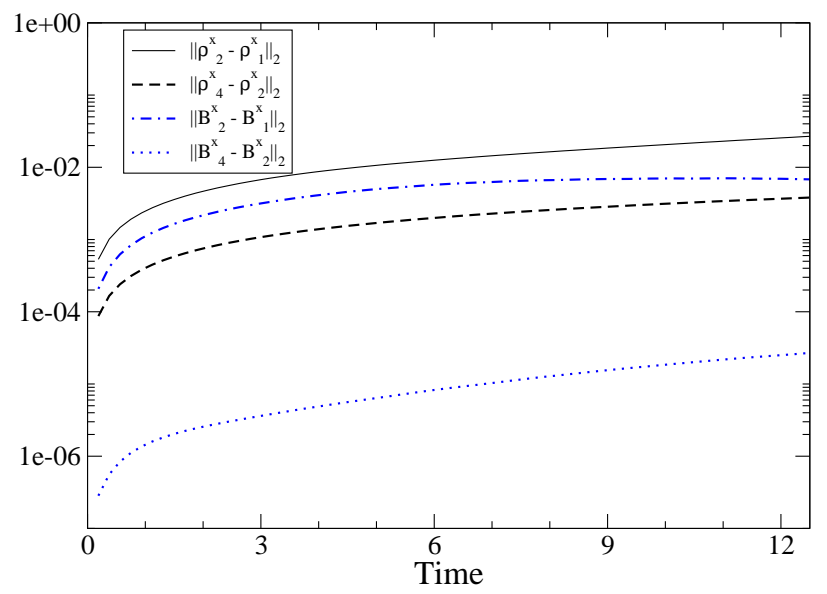

Fig. 1. Behavior of the $L_{2}$ norm of the difference between solutions obtained with gradually improved resolutions. The difference between them converges to zero as expected.

(with no constraint violation or background fluid flow) for three different resolutions $\Delta_{l}=$ $1.5 / 2^{l}(l=0,1,2)$ and check the pair-wise difference of the numerical solutions obtained decreases as expected. Figure 1 illustrates the behavior of $\left\|F\left(\Delta_{l}\right)-F\left(\Delta_{l+1}\right)\right\|_{2}$ (with $F$ either $\rho$ or $\left.B^{x}\right)$. As is evident in the figure, as resolution is improved the differences decrease as expected. For all remaining tests we present results for the finest grid employed with $l=2$.

\subsection{Blast initial data}

In this test, we adopt the blast initial data, evolve it for different choices of boundary conditions setting $\alpha=0, c_{l}=20$ and $s=1$ and examine the constraint's behavior in each case. Figure 2 shows the $L_{2}$ norm of the constraint for the different boundary value conditions. Clearly the numerical solution obtained with constraint preserving boundary conditions is superior by about an order of magnitude in constraint violation than the no-incoming case and almost three orders better than that obtained with the freezing boundary condition. An important point to emphasize is that a closer inspection of the solutions obtained reveals that the main contribution to the error originates at the boundaries in all cases (though with essentially the same behavior as far as the error's magnitude with respect to the boundary condition adopted). The norm displayed in Figure 2 is calculated over the whole computational domain ignoring the last two points at all boundaries to avoid placing excessive weight on the violation at the boundary. Nevertheless, as indicated, constraint preserving boundary conditions give rise to a solution whose constraint is violated the least.

\subsection{Rotor initial data. Effects of boundary conditions and divergence cleaning}

We adopt this data to further examine the solution's behavior and allowing for initial violations of the constraint. To this end we adopt $\kappa=10^{-4}$. 


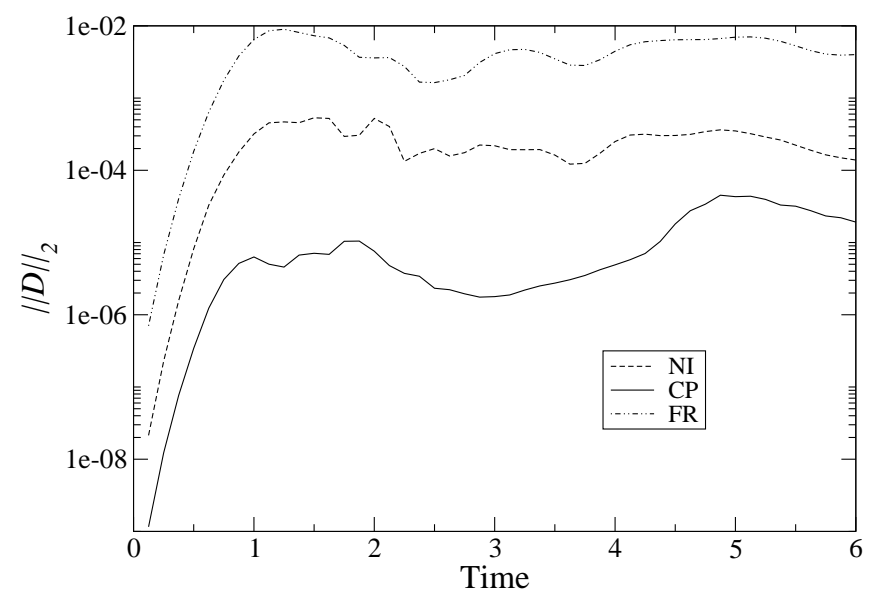

Fig. 2. Behavior of the $L_{2}$ norm of the constraint for the different boundary conditions. The violations in each case grow from round-off values before settling to an approximately constant value. The values throughout the run for this norm improves as the boundary condition adopted is refined as expected, in fact the solution obtained with the constraint preserving boundary condition preserves the constraint by at least three-order of magnitude better than the one obtained with the freezing condition.

Figure 3 illustrates the solution's behavior with the different boundary conditions and with and without the use of the divergence driver. The addition of the divergence driver allows for a dynamical reduction in the constraint violation until $t \simeq 4.6$, at this time the propagating modes interact with the boundaries which become the main source of error. Here again one sees that the constraint preserving boundary condition gives rise to significantly smaller errors in the solution. Interestingly however, adopting the no-incoming modes together with the constraint damping field provides a reasonably similar behavior. This is due to the fact that the no-incoming boundary condition allows the outgoing constraint violating mode to leave the computational domain, while the incoming constraint violating mode, generated by not imposing the constraint preserving boundary condition, is damped to a significant degree by the divergence cleaning technique.

Another interesting behavior is revealed when varying $c_{l}$. This affects the constraint violating modes' propagation speeds which in turn has a strong influence in the solution's constraint behavior. In what follows we adopt the constraint preserving boundary condition. For this test we use the Galilean invariant system, setting $s=0$ (so as not to include damping) and choose $c_{l}$ ranging from 10 to $80 \star$ together with adjusting the time step accordingly in order not to violate the Courant condition. Figures 4 and 5 (with initial constraint violation) show the effect of varying $c_{l}$ in two cases, the Rotor initial data and its modification to include a background velocity given by $\left(\nu^{x}= \pm 0.1 x, \nu^{y}= \pm 0.1 y\right)$. We concentrate on the latter case for there the effect is more striking. Notice that, in figure 4, the initial plateau corresponds to round-off values since the constraint is initially satisfied to that level. Once the non-trivial part of the solution reaches the boundary a significant violation of the constraint is generated.

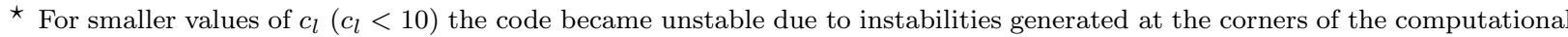
domain.
} 


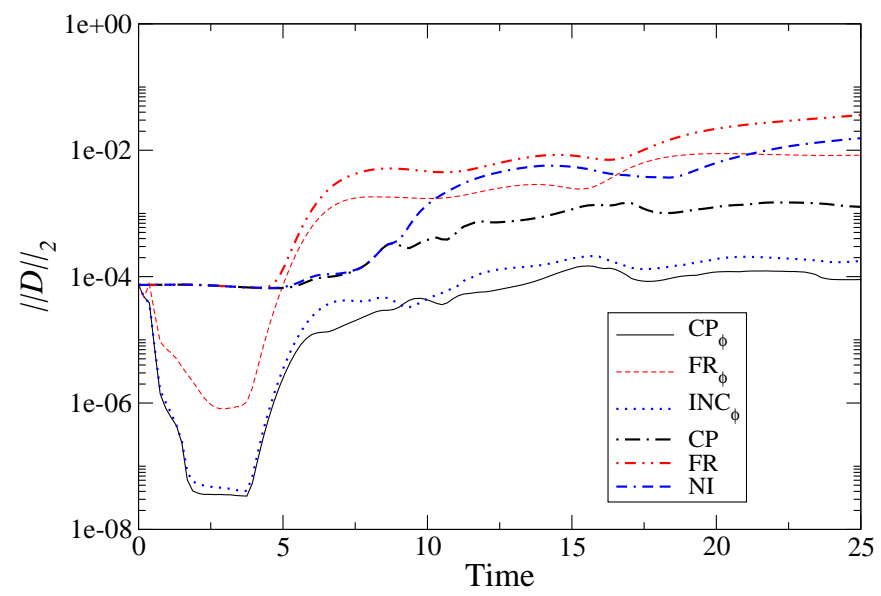

Fig. 3. Behavior of the $L_{2}$ norm of the constraint for different options with some non-trivial initial violation of the constraints. As is evident in the figure, the divergence cleaning is able to damp the constraints by several orders of magnitude while the violation is present in the bulk of the computational domain. After the solution reaches the boundary, the boundary values induced there dominate the violation of the constraint and again the behavior is significantly improved by the no-incoming and constraint preserving conditions.

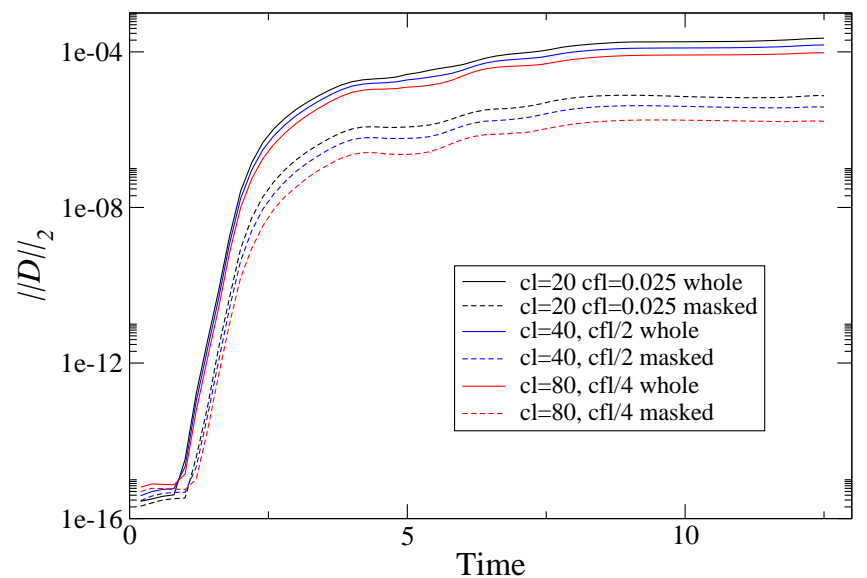

Fig. 4. Behavior of the $L_{2}$ norm of the constraint for different values of the coupling constant $c_{l}$ with constraint preserving boundary condition. The norm is calculated over the whole computational domain (whole) or over an interior region of $3 / 4$ its size (masked). The latter illustrates how, despite not including a region close to the boundaries, boundary induced effects are evident throughout the domain. As the value of $c_{l}$ is increased a slight improvement is achieved.

Depending on the boundary condition, and on the characteristic velocities of the system under consideration that violation propagates to the inside or just leave the domain. In our case, the ability of the constraint preserving boundary condition to allow constraint violating modes to leave the domain, results in smaller errors for faster propagating cases. This is a result of violations induced by the evolution leaved the computational domain more rapidly. 


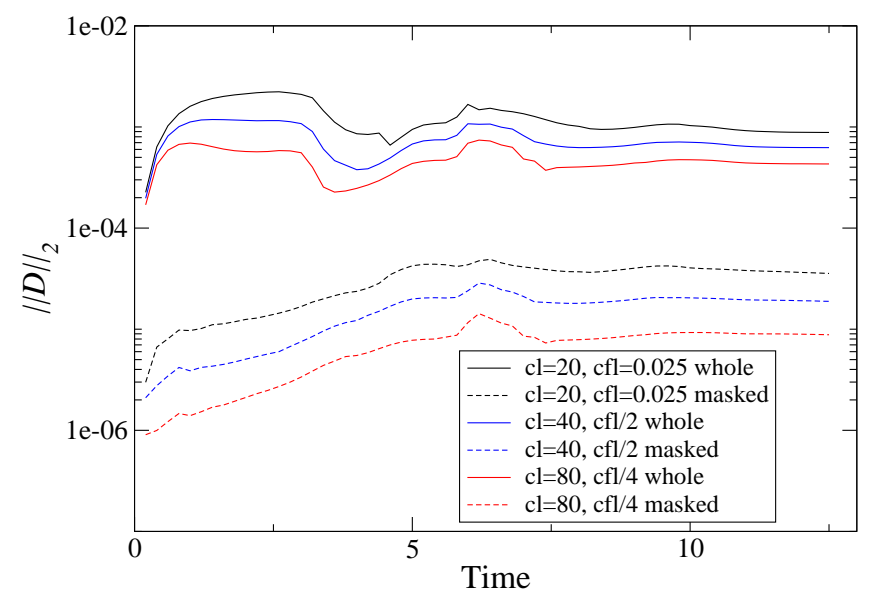

Fig. 5. Similar to figure 4 but with initial data containing constraint violations.

\section{Conclusions}

We have investigated the numerical solution to the (ideal) Newtonian magnetohydrodynamics equations and developed boundary conditions which at the continuum level are constraint preserving and so would not introduce further violations in the computational domain. At the numerical level these conditions do introduce some truncation-error level violations which converge away with resolution. These boundary conditions developed are consistent with maximally dissipative ones and so the discrete energy of the system remains bounded. We also examined the constraint's behavior when enlarging the system so as to couple an extra field. The addition of such field, together with a suitable modification of the equations induces a non-zero speed in the propagation of the constraint and allows for driving its value to zero. We have studied, in particular, two of the many equivalent systems. One which is fully conservative -which is only weakly hyperbolic without the addition of the extra field which renders it symmetric hyperbolic- and a Galilean invariant one which is symmetric hyperbolic -even without adding the extra field- but is not expressible in conservative form.

In order to examine the solution's behavior we implemented the equations in Cartesian coordinates and with a spatial discretization that preserves the initial constraint error. This allows us to separate bulk from boundary effects and observe that the main violations do indeed occur at boundaries. To examine the boundary condition effects on the evolution we adopt three types of boundary conditions:

- The most direct one, and crudest, sets to zero all right hand sides in a buffer boundary region. This might lead to inconsistencies for one might be prescribing conditions to outgoing modes.

This condition is essentially equivalent the most commonly employed one of flux-copying near boundary points.

- The second one is the "no-incoming" boundary condition where one projects out all incoming modes in the evolution equations leaving the rest (tangential and outgoing modes) intact. The implementation of this condition is slightly delicate as modes can change directions over time, and so may turn from being incoming to being outgoing and vice-versa. 
- The third condition preserves the constraint in the sense that is deduced by setting to zero the incoming mode related to the constraint propagation. This condition is a modification

to the previous one where now instead of projecting out all incoming modes, one incoming mode is fixed so that the incoming constraint mode is zero as a result.

Not surprisingly the first option displays the worst behavior for the constraint as not only nothing is done to minimize the constraint violations introduced through the boundaries but further inconsistencies in the solution are induced there. As a result, in the best case the constraint mode bounces back from the boundary into the integration region. The second option performed substantially better displaying a gain of an order of magnitude in constraint preservation. This is a result of the boundary condition allowing for one of the $\phi$ modes carrying constraint violations- to leave the integration region. With the third alternative an additional order of magnitude (at least) is gained with respect to the non-incoming condition. This condition not only allows for constraint violations to leave the computational domain but does not introduce significant violations at the boundary.

Thus we see that appropriately handling the boundary conditions the problem of constraint behavior can be significantly controlled. It should be mentioned that we still do not have a mathematically rigorous proof that the constraint preserving boundary conditions is well posed. However, in simpler systems -like the toy model we discussed at the beginning of the paper- a proof could be devised at least for the case where the eigenvalues do not change sign. Further work in this direction is needed to put the analysis and results obtained in stronger grounds.

\section{Acknowledgments}

We would like to thank M. Anderson, E. Hirschmann, D. Neilsen and C. Palenzuela, for many stimulating discussions during the course of this work. This work was supported by the National Science Foundation under grants PHY-0326311, PHY-0554793, 0653375 to Louisiana State University and PHY05-51164 to the Kavli Institute for Theoretical Physics. L.L. thanks the Kavli Institute for Theoretical Physics for hospitality where parts of this work were completed.

\section{Appendix A. Characteristic decomposition}

In this appendix we revisit the characteristic analysis of the ideal MHD system coupled to the divergence cleaning field (a related discussion in the absence of this field can be found in [30,31]). Using the decomposition on normal and tangential parts, and defining $d:=\sigma+v_{n}$ we get:

$$
\begin{aligned}
d \bar{\rho} & =-\rho \bar{v}_{n}, \\
d \bar{v}_{n} & =2 B_{n} / \rho \bar{B}_{n}-1 / \rho B_{j} \bar{B}^{j}-1 / \rho \bar{p}, \\
d \bar{B}_{n} & =v_{n} \bar{B}_{n}+\bar{\phi} \\
d \bar{p} & =-c_{s}^{2} \rho \bar{v}_{n}-(\gamma-1) B_{j} v^{j} \bar{B}_{n}+(1-\gamma) B_{n} \bar{\phi}, \\
d B \bar{v} & =B^{2} / \rho \bar{B}_{n}-B_{n} / \rho \bar{p}, \\
d B \bar{B} & =B v \bar{B}_{n}-B^{2} \bar{v}_{n}+B_{n} B \bar{v}+B_{n} \bar{\phi}, \\
d \tilde{\bar{v}}_{i} & =\tilde{B}_{i} / \rho \bar{B}_{n}+B_{n} / \rho \tilde{\bar{B}}_{i},
\end{aligned}
$$




$$
\begin{aligned}
d \tilde{\bar{B}}_{i} & =\tilde{v}_{i} \bar{B}_{n}-\tilde{B}_{i} \bar{v}_{n}+B_{n} \tilde{\bar{v}}_{i} . \\
\sigma \bar{\phi} & =c_{l}^{2} \bar{B}_{n}
\end{aligned}
$$

For the sake of clarity we will present the solution to the eigenvalue/eigenvector problem along different cases which are naturally divided by the behavior of certain modes.

\section{A.0.1. BASIS}

- Normal modes. These correspond to $\bar{B}_{n}=\bar{\phi}=0$, where the equations become:

$$
\begin{aligned}
d \bar{\rho} & =-\rho \bar{v}_{n}, \\
d \bar{v}_{n} & =-B \bar{B} / \rho-\bar{p} / \rho, \\
d \bar{p} & =-c_{s}^{2} \rho \bar{v}_{n}, \\
d B \bar{v} & =-\left(B_{n} / \rho\right) \bar{p}, \\
d B \bar{B} & =-B^{2} \bar{v}_{n}+B_{n} B \bar{v}, \\
d \tilde{\bar{v}}_{i} & =\left(B_{n} / \rho\right) \tilde{\bar{B}}_{i}, \\
d \tilde{\bar{B}}_{i} & =-\tilde{B}_{i} \bar{v}_{n}+B_{n} \tilde{\bar{v}}_{i} .
\end{aligned}
$$

Setting $d=0$ one can show that all but the first component must vanish while itself can have any value. So a first eigenvector is given by

$U_{0}=(1,0,0,0,0,0,0)$

where the entries are: $U=\left(\bar{\rho}, \bar{v}_{n}, \tilde{\bar{v}}_{i}, \bar{B}_{n}, \tilde{\bar{B}}_{i}, \bar{p}, \bar{\phi}\right)$.

Using all the equations we get the eigenvalue condition:

$d^{4}-\left(c_{s}^{2}+B^{2} / \rho\right) d^{2}+c_{s}^{2} B_{n}^{2} / \rho=0$.

From which we get four solutions:

$$
\begin{aligned}
& d_{p \pm}= \pm \sqrt{\left(\left(c_{s}^{2}+B^{2} / \rho\right)+\sqrt{\left(c_{s}^{2}+B^{2} / \rho\right)^{2}-4\left(B_{n}^{2} / \rho\right) c_{s}^{2}}\right) / 2} \\
& d_{m \pm}= \pm \sqrt{\left(\left(c_{s}^{2}+B^{2} / \rho\right)-\sqrt{\left(c_{s}^{2}+B^{2} / \rho\right)^{2}-4\left(B_{n}^{2} / \rho\right) c_{s}^{2}}\right) / 2}
\end{aligned}
$$

Notice that when $B^{2} \rightarrow 0$ the $d_{p}$ solutions tend to $\pm c_{s}$, while the $d_{m} \rightarrow 0$ as $\left\|B_{n}\right\| / \sqrt{\rho}$. The $d_{p}$ solutions tend to the fluid solutions while the $d_{m}$ to pure magnetic ones, so they decouple in this limit. One thus must choose the eigenvectors' normalization in a suitable way to reflect this behavior. For the $d_{p}$ solutions we set $\bar{v}_{n}=1$, and use the last two equations to compute the normal magnetic field and the normal velocity, obtaining:

$U P_{ \pm}=\left(\mp \frac{\rho}{d_{p}}, 1,-\frac{B_{n}}{R_{p} \rho} \tilde{B}_{i}, 0, \mp \frac{d_{p}}{R_{p}} \tilde{B}_{i}, \mp \frac{c_{s}^{2} \rho}{d_{p}}, 0\right)$

where $R_{p}:=d_{p}^{2}-B_{n}^{2} / \rho$, has a finite limit as $B^{2} \rightarrow 0$. 
For the $d_{m}$ solutions we proceed the other way around, we fix $B \bar{B}=B_{i} \tilde{\bar{B}}^{i}=B_{i} M^{i}$, where $M_{i}=\tilde{B}_{i} /|\tilde{B}|$ and so compute $\bar{v}_{n}$, and $\bar{p}$ from the second and third equations of system (A.2), obtaining:

$U M_{ \pm}=\left(\frac{B \bar{B}}{R_{m}}, \frac{\mp d_{m} B \bar{B}}{R_{m} \rho}, \frac{ \pm B_{n}}{\rho d_{m}} M_{i}, 0, M_{i}, \frac{c_{s}^{2} B \bar{B}}{R_{m}}, 0\right)$

where $R_{m}:=d_{m}^{2}-c_{s}^{2}$ has a finite limit as $B^{2} \rightarrow 0$.

In two dimensions these are all eigenvalues-eigenvectors pairs in the normal sector.

In three dimensions we can choose $\tilde{\bar{B}}_{i}=M_{i}$ perpendicular to $\tilde{B}_{i}$, but otherwise arbitrary, that is $B \bar{B}=\tilde{B}_{i} \tilde{\bar{B}}^{i}=0$. In this case all other components vanish except the tangential velocity which can be expressed as $\tilde{\bar{v}}_{i}=d_{a} / B_{n} A_{i}$ with $\vec{A}=\left(-\tilde{B}_{2}, \tilde{B}_{1}\right) /|\tilde{B}|$ and $d_{a \pm}= \pm B_{n} / \sqrt{\rho}$ (so that the last two equations can have a nontrivial solution). The corresponding eigenvector is:

$U A_{ \pm}=\left(0,0, \pm \frac{d_{a}}{B_{n}} A_{i}, 0, A_{i}, 0,0\right)$

\section{- $\phi$ modes}

We now look at the modes which are induced by the introduction of the field $\phi$. There, from the third and last equation of (A.1) we get a 2x2 system which implies: $\sigma= \pm c_{l}$ and so, $d:=$ $d_{l}= \pm c_{l}-v_{n}$. If we fix $\bar{B}_{n}=b$ we can obtain the scalar components by solving the following subsystem of (A.1):

$$
\begin{aligned}
d_{l} \bar{v}_{n}+B \bar{B} / \rho+\bar{p} / \rho & =2\left(B_{n} / \rho\right) b, \\
c_{s}^{2} \rho \bar{v}_{n}+\bar{p} d_{l} & =\left(-(\gamma-1) B v+(1-\gamma) \sigma B_{n}\right) b, \\
-B^{2} \bar{v}_{n}-d_{l} B \bar{B}+B_{n} B \bar{v} & =-\left(B_{n} \sigma+B v\right) b, \\
\left(B_{n} / \rho\right) \bar{p}+d_{l} B \bar{v} & =\left(B^{2} / \rho\right) b .
\end{aligned}
$$

From this subsystem we get:

$$
\begin{aligned}
\bar{v}_{n} & =\left[-\left(d_{l}^{2}-B_{n}^{2} / \rho\right) F_{2}+d_{l}^{2} F_{3}+d_{l} \rho\left(d_{l}^{2} F_{1}-\left(B_{n} / \rho\right) F_{4}\right)\right] / \delta_{l}, \\
\bar{p} & =\left(-c_{s}^{2} \rho \bar{v}_{n}+F_{2}\right) / d_{l},
\end{aligned}
$$

where:

$$
\begin{aligned}
\delta_{l} & =\rho\left(d_{l}^{4}-d_{l}^{2}\left(c_{s}^{2}+B^{2} / \rho\right)+c_{s}^{2} B_{n}^{2} / \rho\right), \\
F_{1} & =\frac{-2 B_{n} b}{\rho}, \\
F_{2} & =(1-\gamma)\left(B v+B_{n} \sigma\right) b, \\
F_{3} & =-F_{2} /(1-\gamma), \\
F_{4} & =\frac{B^{2} b}{\rho} .
\end{aligned}
$$

Once we have $\bar{v}_{n}$ we can solve for the vectorial components of the system: 


$$
\begin{aligned}
d_{l} \tilde{\bar{v}}_{i}-\left(B_{n} / \rho\right) \tilde{\bar{B}}_{i} & =\tilde{B}_{i} b / \rho, \\
d_{l} \tilde{\bar{B}}_{i}-B_{n} \tilde{\bar{v}}_{i} & =\tilde{v}_{i} b-\tilde{B}_{i} \bar{v}_{n},
\end{aligned}
$$

obtaining:

$$
\begin{aligned}
\tilde{\bar{v}}_{i} & =\left[\tilde{v}_{i} b B_{n} / \rho+\tilde{B}_{i}\left(d_{l} b-\bar{v}_{n} B_{n}\right) / \rho\right] / \delta_{s}, \\
\tilde{\bar{B}}_{i} & =\left[\tilde{v}_{i} d_{l} b+\tilde{B}_{i}\left(-d_{l} \bar{v}_{n},+b B_{n}^{2} / \rho\right)\right] / \delta_{s},
\end{aligned}
$$

where $\delta_{s}:=d_{l}^{2}-B_{n}^{2} / \rho$. Combining the above intermediate steps we finally obtain,

$$
\begin{aligned}
U L_{ \pm}= & \left(-\left(\rho / d_{l}\right) \bar{v}_{n},\left[-\left(d_{l}^{2}-B_{n}^{2} / \rho\right) F_{2}+d_{l}^{2} F_{3}+d_{l} \rho\left(d_{l}^{2} F_{1}-\frac{B_{n} F_{4}}{\rho}\right)\right] / \delta_{l},\right. \\
& {\left[\tilde{v}_{i} b B_{n} / \rho+\tilde{B}_{i}\left(d_{l} b-\bar{v}_{n} B_{n}\right) / \rho\right] / \delta_{s}, b,\left[\tilde{v}_{i} d_{l} b+\tilde{B}_{i}\left(-d_{l} \bar{v}_{n}+\frac{b B_{n}^{2}}{\rho}\right)\right] / \delta_{s} } \\
& \left.\frac{\left(-c_{s}^{2} \rho \bar{v}_{n}+F_{2}\right.}{d_{l}}, b c_{l}^{2} / \sigma\right)
\end{aligned}
$$

\section{A.0.2. $C O-B A S I S$}

Net we compute the co-basis which we will use to construct the suitable projector for enforcing different boundary conditions. As in the previous analysis, we divide our task along different eigenvalues for simplicity.

- $\Theta_{0}$

Since $U_{0}$ has only one non-vanishing component (the first one) all elements of the co-base, except the corresponding one have a vanishing first element.

The first element, is simply:

$\Theta_{0}=\left(1,0,0, A, 0,-c_{s}^{-2}, B\right)$.

To find the remaining elements we notice that if we define: $V P:=\left(U L_{+}+U L_{-}\right)$and $V M:=$ $\left(U L_{+}-U L_{-}\right)$, the first has a zero in the last component (corresponding to $\bar{\phi}$ ) while the second has a zero in the fourth component (corresponding to $\bar{B}_{n}$ ). Thus contraction of $\Theta_{0}$ with $V P$ and $V M$ will leave either $A$ or $B$ only as unknowns. Thus we might proceed to setting $A$ and $B$ temporarily to zero, perform the contraction and extract what these must be obtaining,

$$
\begin{aligned}
& A=\frac{-\Theta_{0}(V P)}{2 b}, \\
& B=\frac{-\Theta_{0}(V M)}{2 b c_{l}},
\end{aligned}
$$

where we first temporarily set to zero $A$, and $B$ in $\Theta_{0}$.

- $\Theta_{P \pm}$

From 
$\Theta_{P \pm}\left(U M_{+}-U M_{-}\right)=0$ and

$\Theta_{P \pm}\left(U M_{+}+U M_{-}\right)=0$

we get that the following structure for it:

$\Theta_{P \pm}=\left(0, C, C \frac{d_{m}^{2} \tilde{B}^{i}}{B_{n} R_{m}}, D_{ \pm}, \frac{-E_{ \pm} c_{s}^{2} \tilde{B}^{i}}{R_{m}}, E_{ \pm}, F_{ \pm}\right)$.

Now, from $1=\Theta_{P \pm}\left(U P_{+}+U P_{-}\right)$we obtain:

$C=\frac{R_{p} R_{m}}{2\left(R_{p} R_{m}-d_{m}^{2} \tilde{B}^{2} / \rho\right)} \quad$ (The same for both).

While from $\pm 1=\Theta_{P \pm}\left(U P_{+}-U P_{-}\right)$we deduce

$E_{ \pm}=\frac{ \pm R_{p} R_{m} d_{p}}{2 c_{s}^{2}\left(d_{p}^{2} \tilde{B}^{2}-\rho R_{p} R_{m}\right)}$

The remaining two components can be computed using $V P$ and $V M$ as above obtaining,

$D_{ \pm}=\frac{-\Theta_{P \pm}(V P)}{2 b}$,
$F_{ \pm}=\frac{-\Theta_{P \pm}(V M)}{2 b c_{l}}$,

where we set to zero $D$, and $F$ in $\Theta_{P \pm}$, as in the previous case.

- $\Theta_{M \pm}$

From $\Theta_{M \pm}\left(U P_{+}-U P_{-}\right)=0$ and $\Theta_{M \pm}\left(U P_{+}+U P_{-}\right)=0$ we get that the following structure for it:

$\Theta_{M \pm}=\left(0, \frac{B_{n} \tilde{B}_{i} N_{ \pm}^{i}}{\rho R_{p}}, N_{ \pm}^{i}, S_{ \pm}, L^{i}, \frac{-d_{p}^{2} \tilde{B}_{i} L^{i}}{c_{s}^{2} R_{p} \rho}, T_{ \pm}\right)$.

From $1=\Theta_{M \pm}\left(U M_{+}+U M_{-}\right)$we obtain:

$L^{i}=-\frac{R_{m} R_{p} M^{i}}{2\left(\tilde{B}^{2} d_{p}^{2} / \rho-R_{m} R_{p}\right)}$.

From $\pm 1=\Theta_{M \pm}\left(U M_{+}-U M_{-}\right)$we obtain:

$N_{ \pm}^{i}=\mp \frac{d_{m} R_{m} R_{p} M^{i}}{2 B_{n} / \rho\left(\tilde{B}^{2} d_{m}^{2} / \rho-R_{m} R_{p}\right)}$.

The remaining two components, $S$ and $T$ are computed as in the previous case, using: $V P$ and $V M$ removing the $\bar{B}_{n}$ and the last component $\bar{\phi}$ in $\Theta_{M \pm}$. We get,

$S_{ \pm}=\frac{-\Theta_{M \pm}(V P)}{2 b}$,
$T_{ \pm}=\frac{-\Theta_{M \pm}(V M)}{2 b c_{l}}$. 
- $\Theta_{A \pm}$

We get the co-vector of $U A$ by direct computation:

$\Theta_{A \pm}=\left(0,0, \pm \frac{B_{n} A^{i}}{2 d_{a}}, G_{ \pm}, \frac{A^{i}}{2}, 0, H_{ \pm}\right)$

where the components, $G$ and $H$ are given by:

$$
\begin{aligned}
& G_{ \pm}=\frac{-\Theta_{A \pm}(V P)}{2 b}, \\
& H_{ \pm}=\frac{-\Theta_{A \pm}(V M)}{2 b c_{l}} .
\end{aligned}
$$

removing the components $\bar{B}_{n}$ and $\bar{\phi}$ in $\Theta_{A \pm}$.

- $\Theta_{L \pm}$

It remains now to determine the last two elements. Since the first 7 eigenvectors span completely the seven dimensional space given by $\left(\bar{B}_{n}=\bar{\phi}=0\right)$ the co-vectors have only components in that subspace and are given by:

$\Theta_{L \pm}=\left(0,0,0, \frac{1}{2 b}, 0,0, \frac{ \pm 1}{2 b c_{l}}\right)$.

\section{Caveat: Singular points}

The characteristic structure outlined above may change as some eigenvalues can change multiplicity for particular values of the fields. These special cases require further analysis. Recall the eigenvalues are given by:

$$
\begin{aligned}
\sigma_{0} & =-v_{n}, \\
\sigma_{P}^{+} & =d_{p}-v_{n}, \\
\sigma_{P}^{-} & =-d_{p}-v_{n}, \\
\sigma_{M}^{+} & =d_{m}-v_{n}, \\
\sigma_{M}^{-} & =-d_{m}-v_{n}, \\
\sigma_{A}^{+} & =d_{a}-v_{n}, \\
\sigma_{A}^{-} & =-d_{a}-v_{n}, \\
\sigma_{L}^{+} & =c_{l}, \\
\sigma_{L}^{-} & =-c_{l},
\end{aligned}
$$

they can cross when $d_{p}=0, v_{n}= \pm c_{l}, d_{m}=0$ and $d_{p}=d_{m}$. The first two cases can be dealt with by appropriately chosen parameters so that they would not occur in physically relevant scenarios. For instance, the first case would imply a vanishing sound speed and will not be considered since we will not deal with a fluid describing dust. The second case could be avoided by simply taking $c_{l}$ large enough. Therefore, we concentrate on the other two:

$$
\text { - } d_{m}=0
$$


For this we need that

$$
\left(c_{s}^{2}+\frac{B^{2}}{\rho}\right)^{2}=\left(c_{s}^{2}+\frac{B^{2}}{\rho}\right)^{2}-4 c_{s}^{2} \frac{B_{n}^{2}}{\rho}
$$

which only happens when $B_{n}=0$. Near that point we have,

$d_{m}^{2}=\frac{c_{s}^{2} B_{n}^{2}}{\rho c_{s}^{2}+B^{2}}$.

At this point we compute explicitly the eigenspace for the coinciding eigenvalues and choose eigenvectors with good limit. We use them in a sufficiently small neighborhood of this point using conditional statements in the code at every point in the boundary.

In three dimensions, in this case, also $d_{a}=0$, therefore the eigenvectors $U M_{ \pm}$and $U A_{ \pm}$are degenerates.

- $d_{m}=d_{p}$

For this to happen we need

$0=\left(d_{p}^{2}-d_{m}^{2}\right)^{2}=\left(c_{s}^{2}+\frac{B^{2}}{\rho}\right)^{2}-4 c_{s}^{2} B_{n}^{2}=\left(c_{s}^{2}-\frac{B_{n}^{2}}{\rho}\right)^{2}+2 c_{s}^{2} \frac{\tilde{B}_{i}^{2}}{\rho}+\frac{\tilde{B}_{i}^{4}}{\rho^{2}}$.

Thus. $B_{n}^{2} / \rho=c_{s}^{2}$, and $\tilde{B}_{i}=0$. Notice that in that case we have $R_{p}=R_{m}=0$. We proceed in a similar fashion as above. Notice that when eigenvalues coincide all what enters in the boundary condition is the projector on that subspace, so one just has to choose the eigenvectors so that numerically that projector is robust in a small neighborhood.

In the case of three dimensions, $d_{a}^{2}=B_{n}^{2} / \rho$. Hence, $d_{a}=d_{m}=d_{p}$ and the eigenvectors $U P_{ \pm}$, $U M_{ \pm}$and $U A_{ \pm}$become degenerates.

\section{References}

[1] H. Goedbloed and S. Poedts, "Principles of Magnetohydrodynamics". Cambridge Univ. Press, Cambridge, 2004.

[2] P.A. Davidson, "An introduction to Magnetohydrodynamics". Cambridge Univ. Press, Cambridge, 2001.

[3] J. F. Hawley and C. Evans, "Simulation of mhd flows: A constrained transport method," Astrophys., vol. 332, p. 659 , 1988.

[4] G. Toth, "The $\nabla B$ constraint in Schock-Capturing Magnetohydrodynamics", J. Comp. Phys. 161, 605-652 (2004).

[5] C. F. Gammie, J. C. McKinney and G. Toth, Astrophys. J. 589, 444 (2003)

[6] T. A. Gardiner and J. M. Stone, "An unsplit godunov method for ideal mhd via constrained transport," J. Comput. Phys., vol. 205, pp. 509-539, 2005.

[7] S. A. Balbus and J. F. Hawley, "Numerical simulations of mhd turbulence in accretion disks," Lect. Notes Phys., vol. 614, pp. 329-350, 2003.

[8] M. Shibata and Y.-i. Sekiguchi, "Magnetohydrodynamics in full general relativity: Formulation and tests," Phys. Rev., vol. D72, p. 044014, 2005.

[9] M. D. Duez, Y. T. Liu, S. L. Shapiro, and B. C. Stephens, "Relativistic magnetohydrodynamics in dynamical spacetimes: Numerical methods and tests," Phys. Rev., vol. D72, p. 024028, 2005.

[10] B. Giacomazzo and L. Rezzolla, "Whiskymhd: a new numerical code for general relativistic magnetohydrodynamics," Class. Quant. Grav., vol. 24, pp. S235-S258, 2007.

[11] S. Li, H. Li and R. Cen, arXiv:astro-ph/0611863

[12] M. Obergaulinger, M. A. Aloy and E. Muller, Astron. Astrophys. 450, 1107 (2006)

[13] J.S. Kim, D. Ryu, T.W. Jones and S.S. Hong, Astrophysical J. 514, 506 (1999). 
[14] A Dedner, F Kemm, D Kröner, C-D Munz, T Schnitzer, and M Wesenberg. Hyperbolic divergence cleaning for the MHD equations. J. Comput. Phys., 175:645, 2002.

[15] D.S. Balsara and J.S. Kim, Astrophys. J. 602, 1079 (2004).

[16] M. Anderson, E. Hirschmann, S. L. Liebling and D. Neilsen, Class. Quant. Grav. 23, 6503 (2006).

[17] D. Neilsen, E. W. Hirschmann and R. S. Millward, Class. Quant. Grav. 23, S505 (2006)

[18] A. Dedner, D. Kroner, I. Sofronov and W. Wesenberg, J. Comput. Phys. 171, 448 (2001).

[19] R. Leveque, "Finite Volume Methods for Hyperbolic Problems", Cambridge University Press, 2002.

[20] A. Zachary, A. Malagoli and P. Colella, SCIAM J. Sci. Comput, 15, 263 (1994).

[21] D. Balsara, Astrop. J. Supp. 116, 119 (1998).

[22] D. De Zeeuw, T. Gombosi, C. Groth, K. Powerl and Q. Stout, IEEE Trans. Plasma Science, 28, 1956 (2000).

[23] H. Yee and B. Sjogreen, in Proceedins of the International Conference on High Performance Scientific Computing, Hanoi, Vietnam. (2003). Cambridge University Press, 2002.

[24] B. Gustaffson, H. Kreiss, and J. Oliger, Time Dependent Problems and Difference Methods (Wiley, New York, 1995).

[25] Oscar A Reula, Journal of Hyperbolic Differential Equations 1:22, 251-269, 6/2004

[26] S. K. Godunov, The symmetric form of magnetohydrodynamics equation, Numer. Methods Mech. Contin. Media 1,26 (1972).

[27] T. J. Barth, Numerical methods for gasdynamic systems on unstructured meshes, in An Introduction to Recent Developments in Theory and Numerics for Conservation Laws: Proceedings of the International School, Freiburg/Littenweiler, Germany, October 20?24, 1997, edited by D. Kröner, M. Ohlberger, and C. Rohde, Lecture Notes in Computational Science and Engineering (Springer-Verlag, Berlin, 1999), Vol. 5, p. 195.

[28] D. Balsara and D. Spicer, Journal of Comp. Phys., 148 133, (1999).

[29] L. Del Zanna, N. Bucciantini and P. Lodrillo, Astron. Astrophys. 400, 397, (2003).

[30] S. Pennisi, "A covariant and extended model for relativistic magnetofluiddynamics". Ann. Inst. Henri Poincare 58, 343-361 (1993).

[31] D. Balsara, "Total variation diminishing for relativistic magnetohydrodynamics". The Astrophysical Journal Supp. Series, 132, 83-101 (2001). 